\title{
OPEN PROBLEMS AND CONJECTURES ON RATIONAL SYSTEMS IN THREE DIMENSIONS
}

\author{
G. LADAS, G. LUGO AND F. J. PALLADINO
}

Dedicated to Professor Mustafa Kulenovic on the occasion of his $60^{\text {th }}$ birthday

\begin{abstract}
We present some open problems and conjectures on Rational Systems in three dimensions, or higher, with nonnegative parameters and with nonnegative initial conditions such that the denominators are always positive. We also employ the method of Full Limiting Sequences to confirm an outstanding conjecture on $k^{\text {th }}$-order rational difference equations.
\end{abstract}

\section{INTRODUCTION}

In this paper, we present some open problems and conjectures on rational systems in three dimensions of the form:

$$
\left.\begin{array}{l}
x_{n+1}=\frac{\alpha_{1}+\beta_{1} x_{n}+\gamma_{1} y_{n}+\delta_{1} z_{n}}{A_{1}+B_{1} x_{n}+C_{1} y_{n}+D_{1} z_{n}} \\
y_{n+1}=\frac{\alpha_{2}+\beta_{2} x_{n}+\gamma_{2} y_{n}+\delta_{2} z_{n}}{A_{2}+B_{2} x_{n}+C_{2} y_{n}+D_{2} z_{n}} \\
z_{n+1}=\frac{\alpha_{3}+\beta_{3} x_{n}+\gamma_{3} y_{n}+\delta_{3} z_{n}}{A_{3}+B_{3} x_{n}+C_{3} y_{n}+D_{3} z_{n}}
\end{array}\right\}, \quad n=0,1, \ldots
$$

with nonnegative parameters and with nonnegative initial conditions such that the denominators are always positive.

We also employ the method of Full Limiting sequences, see Theorem 1.8 in [27], to confirm Conjecture 1, in Section 2, about the global character of

2000 Mathematics Subject Classification. 39A10, 39A11.

Key words and phrases. Difference equation, global asymptotic stability, boundedness character, difference inequality, rational system. 
solutions of the $(k+1)^{t h}$-order rational difference equation :

$$
x_{n+1}=\frac{\alpha}{1+\prod_{i=0}^{k} x_{n-i}}, \quad n=0,1, \ldots
$$

which is derived from a rational system in higher dimensions.

A systematic work on the global character of rational systems in two dimensions of the form :

$$
\left.\begin{array}{l}
x_{n+1}=\frac{\alpha_{1}+\beta_{1} x_{n}+\gamma_{1} y_{n}}{A_{1}+B_{1} x_{n}+C_{1} y_{n}} \\
y_{n+1}=\frac{\alpha_{2}+\beta_{2} x_{n}+\gamma_{2} y_{n}}{A_{2}+B_{2} x_{n}+C_{2} y_{n}}
\end{array}\right\}, \quad n=0,1, \ldots
$$

was intitiated in [15]. Several special cases of system (3) have been investigated by Kulenović and Merino and their students, and also by Ladas and his collaborators and students. See [1]-[3], [5]-[7], [9]-[21], [25], [28]-[29], [31]-[40]. See also: [4], [8], [22]-[23], [26], [30], [41]-[42], and [45].

It is an amazing fact that system (1) contains,

$\left(2^{4}-1\right) \times\left(2^{4}-1\right) \times\left(2^{4}-1\right) \times\left(2^{4}-1\right) \times\left(2^{4}-1\right) \times\left(2^{4}-1\right)=11,390,625$

special cases of rational systems in 3 dimensions and it is of paramount importance to understand the global character of solutions of each one of these special cases.

\section{Open PRoblems AND CONJECTURES}

Here we pose some open problems and conjectures on the global character of solutions of System (1).

We wish to determine the boundedness characterization of each special case of System (1). In the past work on systems of two rational difference equations, patterns emerged eventually after a large amount of work describing the boundedness characterizations on a case by case basis. We are especially interested in finding similar patterns of boundedness for System (1).

For each system with bounded solutions we wish to determine the global stability character of their equilibrium points and the periodic nature of their solutions.

For each system with unbounded solutions, we wish to determine the way that their solutions are unbounded, the stable and unstable manifolds of their equilibrium points, any invariants and whether there exists any periodic trichotomies. 
We now pose an open problem for the simpler system :

$$
\left.\begin{array}{c}
x_{n+1}=\frac{\alpha_{1}}{y_{n}} \\
y_{n+1}=\frac{\alpha_{2}}{z_{n}} \\
z_{n+1}=\frac{\alpha_{3}+\beta_{3} x_{n}+\gamma_{3} y_{n}+\delta_{3} z_{n}}{A_{3}+B_{3} x_{n}+C_{3} y_{n}+D_{3} z_{n}}
\end{array}\right\}, \quad n=0,1, \ldots
$$

System (4) contains 225 of the 11,390,625 rational systems included in System (1).

By eliminating the variables $x_{n}$ and $y_{n}$ from the third equation in (4), we see that $\left\{z_{n}\right\}$ satisfies a third order rational difference equation of the form:

$$
z_{n+1}=\frac{\gamma+\alpha z_{n-1}+\delta z_{n} z_{n-1}+\alpha_{1} \beta z_{n-1} z_{n-2}}{C+A z_{n-1}+D z_{n} z_{n-1}+\alpha_{1} B z_{n-1} z_{n-2}}, \quad n=0,1, \ldots
$$

with nonnegative parameters and nonnegative initial conditions such that the denominators are always positive.

Open Problem 1. Investigate the global character of solutions of the 225 special cases which are included in Eq. (5). For each special case, determine its boundedness character, its periodic behavior, and the global stability character of its equilibrium points.

The following conjectures deal with some higher order analogues of System (4) and equation (5).

Assume $\alpha>0$. Then we pose the following three conjectures :

Conjecture 1. Every positive solution of the difference equation:

$$
z_{n+1}=\frac{\alpha}{1+\prod_{i=0}^{k} z_{n-i}}, \quad n=0,1, \ldots
$$

has a finite limit.

This result was established, in [24], for the case in which $k=1$.

Conjecture 2. Every positive solution of the difference equation:

$$
z_{n+1}=\frac{\alpha}{1+\prod_{i=1}^{k} z_{n-i}}, \quad n=0,1, \ldots
$$

converges to a (not necessarily prime) period $(k+3)$ solution.

Conjecture 3. Every positive solution of the difference equation:

$$
z_{n+1}=\frac{\alpha}{1+z_{n-l} z_{n-k}}, \quad n=0,1, \ldots
$$


converges to a (not necessarily prime) period $(l+k+2)$ solution.

We now present some other conjectures about special cases of System (1), which are included in the following simpler system :

$$
\left.\begin{array}{r}
x_{n+1}=\gamma_{1} y_{n} \\
y_{n+1}=\delta_{2} z_{n} \\
z_{n+1}=\frac{\alpha_{3}+\beta_{3} x_{n}+\gamma_{3} y_{n}+\delta_{3} z_{n}}{A_{3}+B_{3} x_{n}+C_{3} y_{n}+D_{3} z_{n}}
\end{array}\right\}, \quad n=0,1, \ldots
$$

This system reduces to the following third order rational difference equation :

$$
z_{n+1}=\frac{\alpha+\beta z_{n}+\gamma z_{n-1}+\delta z_{n-2}}{A+B z_{n}+C z_{n-1}+D z_{n-2}}, \quad n=0,1, \ldots,
$$

This equation was investigated in [16]. In [16], the authors posed several interesting open problems and conjectures on the difference equation (7). There are several noteworthy conjectures, which have not yet been resolved.

Conjecture 4. Assume $C>0$ and that an equilibrium point $\overline{\mathrm{z}}$ of the difference equation

$$
z_{n+1}=\frac{\alpha+\beta z_{n}+\gamma z_{n-1}}{A+B z_{n}+C z_{n-1}}, \quad n=0,1, \ldots,
$$

is locally asymptotically stable. Show that $\overline{\mathrm{x}}$ is a global attractor of all positive solutions of equation (8).

To prove Conjecture 4, it is necessary that following conjecture be confirmed.

Conjecture 5. For the difference equation

$$
z_{n+1}=\frac{\alpha+\beta z_{n}}{A+z_{n}+C z_{n-1}}, \quad n=0,1, \ldots
$$

assume $A \geq 0$, all other parameters positive, and nonnegative initial conditions such that the denominators are never zero. Show that every solution of (9) converges.

For the most recent account of the progress made on Conjecture 4, see [5], [16], [41] and [46].

Another interesting conjecture is the following period-six trichotomy conjecture : 
Conjecture 6. Assume that $\alpha, C \in[0, \infty)$. Then the following period-six trichotomy result is true for the rational equation

$$
z_{n+1}=\frac{\alpha+z_{n}}{C z_{n-1}+z_{n-2}}, \quad n=0,1, \ldots
$$

(a) Every positive solution of equation (10) converges to its positive equilibrium, if and only if, $\alpha C^{2}>1$.

(b) Every positive solution of equation (10) converges to a not necessarily prime period-six solution of equation (10), if and only if, $\alpha C^{2}=1$.

(c) Equation (10) has positive unbounded solutions, if and only if, $\alpha C^{2}<$ 1.

The only part of this conjecture which has been resolved is the case for which $\alpha C^{2}=0$. This was resolved in [43].

Related to Conjecture 8 is the following conjecture :

Conjecture 7. For the difference equation

$$
z_{n+1}=\frac{\alpha+z_{n}}{C z_{n-1}+z_{n-2}}, \quad n=0,1, \ldots
$$

assume positive parameters and nonnegative initial conditions such that the denominators are never zero. The difference equation (11), has unbounded solutions in some range of the parameters.

This is the only special case of (7) whose boundedness character has not been established yet. We present another conjecture about the boundedness character of (7).

Conjecture 8. Assume $\alpha, \beta, \gamma \in[0, \infty)$. Then every positive solution of the difference equation

$$
z_{n+1}=\frac{\alpha+\beta z_{n}+\gamma z_{n-1}}{z_{n-2}}, \quad n=0,1, \ldots
$$

is bounded, if and only if, $\beta=\gamma$.

For the most recent work on the boundedness character of (7) see [16], [43] and [44].

\section{Confirmation of Conjecture 1}

In this section, we establish the following theorem, which confirms the above Conjecture 1 .

Theorem 1. Every solution of the $(k+1)^{\text {th }}$-order difference equation

$$
x_{n+1}=\frac{\alpha}{1+\prod_{i=0}^{k} x_{n-i}}, \quad n=0,1, \ldots,
$$

has a finite limit. 
For the proof of this theorem we need the following lemma, which is Theorem 1.8 of [27].

Lemma 1. Consider the difference equation

$$
x_{n+1}=f\left(x_{n}, x_{n-1}, \ldots, x_{n-k}\right),
$$

where $f \in C\left[J^{k+1}, J\right]$ for some interval of real numbers $J$ and some nonnegative integer $k$. Let $\left\{x_{n}\right\}_{n=-k}^{\infty}$ be a solution of (14). Set $I=\lim _{n \rightarrow \infty} \inf x_{n}$ and $S=\lim _{n \rightarrow \infty} \sup x_{n}$, and suppose that $I, S \in J$. Let $\mathcal{L}_{0}$ be a limit point of the sequence $\left\{x_{n}\right\}_{n=-k}^{\infty}$. Then the following statements are true:

1. There exists a solution $\left\{L_{n}\right\}_{n=-\infty}^{\infty}$ of (14), called a full limiting sequence of $\left\{x_{n}\right\}_{n=-k}^{\infty}$, such that $L_{0}=\mathcal{L}_{0}$, and such that for every $N \in\{\ldots,-1,0,1, \ldots\}, L_{N}$ is a limit point of $\left\{x_{n}\right\}_{n=-k}^{\infty}$. In particular,

$$
I \leq L_{N} \leq S \quad \text { for all } \quad N \in\{\ldots,-1,0,1, \ldots\} .
$$

2. For every $i_{0} \in\{\ldots,-1,0,1, \ldots\}$, there exists a subsequence $\left\{x_{r_{i}}\right\}_{i=0}^{\infty}$ of $\left\{x_{n}\right\}_{n=-m}^{\infty}$ such that

$$
L_{N}=\lim _{i \rightarrow \infty} x_{r_{i}+N} \quad \text { for all } \quad N \geq i_{0} .
$$

The result that every solution of the difference equation (13) has a finite limit will follow as a corollary of the following theorem.

Theorem 2. Every solution of the $(k+2)$-order difference equation

$$
x_{n+1}=\frac{x_{n}\left(1+\prod_{i=1}^{k+1} x_{n-i}\right)}{1+\prod_{i=0}^{k} x_{n-i}}, \quad n=0,1, \ldots,
$$

has a finite limit.

This is because every solution of the difference equation (13) converges if every solution of difference equation (15) converges. This is true because every solution of (13) is a solution of (15). This will be demonstrated below in the case for which $k=2$.

For simplicity of the presentation, we will just establish Theorem 1 for the third order difference equation,

$$
x_{n+1}=\frac{\alpha}{1+x_{n} x_{n-1} x_{n-2}}, \quad n=0,1, \ldots
$$

The proof for the general case is similar but lengthier.

Notice that, for the difference equation (16), we have

$$
x_{n}\left(1+x_{n-1} x_{n-2} x_{n-3}\right)=\alpha, \quad n=1,2, \ldots
$$


Using (17), we can embed the difference equation (16) into the difference equation

$$
x_{n+1}=\frac{x_{n}\left(1+x_{n-1} x_{n-2} x_{n-3}\right)}{1+x_{n} x_{n-1} x_{n-2}}, \quad n=1,2, \ldots
$$

which is a special case of the difference equation (15).

To establish Theorem 2 , for the case where $k=2$, we need the following lemma.

Lemma 2. Let $\left\{x_{n}\right\}_{n=-2}^{\infty}$ be a solution of (18). Set

$$
m=\min \left\{x_{-2}, x_{-1}, x_{0}, x_{1}\right\}
$$

and

$$
M=\max \left\{x_{-2}, x_{-1}, x_{0}, x_{1}\right\} .
$$

Then

$$
m \leq x_{n} \leq M, \text { for all } n \geq-2 .
$$

Proof. Clearly

$$
m \leq x_{n} \leq M, \quad \text { for all } \quad-2 \leq n \leq 1 .
$$

Notice that

$$
x_{2}=\frac{x_{1}\left(1+x_{0} x_{-1} x_{-2}\right)}{1+x_{1} x_{0} x_{-1}} .
$$

and

and

$$
\frac{\partial}{\partial w}\left(\frac{w(1+x y z)}{1+w x y}\right)=\frac{x y z+1}{(w x y+1)^{2}}>0
$$

$$
\frac{\partial}{\partial z}\left(\frac{w(1+x y z)}{1+w x y}\right)=\frac{w x y}{w x y+1}>0 .
$$

By (19) and (20) it follows that

$$
m=\frac{m\left(1+x_{0} x_{-1} m\right)}{1+m x_{0} x_{-1}} \leq \frac{x_{1}\left(1+x_{0} x_{-1} x_{-2}\right)}{1+x_{1} x_{0} x_{-1}} \leq \frac{M\left(1+x_{0} x_{-1} M\right)}{1+M x_{0} x_{-1}}=M .
$$

and by induction, that

$$
m \leq x_{n} \leq M \text { for all } n \geq-2 .
$$

To complete the proof, it suffices to show that

$$
\lim _{n \rightarrow \infty} \inf x_{n}=\lim _{n \rightarrow \infty} \sup x_{n} .
$$

For the sake of contradiction, assume that

$$
\lim _{n \rightarrow \infty} \inf x_{n}<\lim _{n \rightarrow \infty} \sup x_{n} .
$$


By part 1. of Lemma 1, there exists a full limiting sequence $\left\{L_{n}\right\}_{n=-\infty}^{\infty}$ of $\left\{x_{n}\right\}_{n=-2}^{\infty}$ with $L_{0}=I=\lim _{n \rightarrow \infty} \inf x_{n}$.

We claim that $L_{n}=I$ for all $n \in \mathbb{Z}$.

We first show that $L_{-n}=L_{0}$ for all $n=0,1, \ldots$ We will show $L_{-1}=L_{0}$. For the sake of contradiction, assume that $L_{-1}>L_{0}$. Then, by (19) and (20), (18) is increasing with respect to the first and last argument. Hence

$$
L_{0}=\frac{L_{-1}\left(1+L_{-2} L_{-3} L_{-4}\right)}{1+L_{-1} L_{-2} L_{-3}}>\frac{L_{0}\left(1+L_{-2} L_{-3} L_{0}\right)}{1+L_{0} L_{-2} L_{-3}}=L_{0} .
$$

This is a contradiction. It follows by induction that $L_{-n}=L_{0}$ for all $n=$ $0,1, \ldots$

It follows that $L_{n}=I$, for all $n \in \mathbb{N}$, since every point of (18) is an equilibrium point and since $L_{-n}=L_{0}$ for all $n=0,1, \ldots$

By part 2 of Lemma 1, there exists a subsequence $\left\{x_{r_{i}}\right\}_{i=0}^{\infty}$ of $\left\{x_{n}\right\}_{n=-2}^{\infty}$ such that

$$
\lim _{i \rightarrow \infty} x_{r_{i-j}}=L_{-j}
$$

for every $-1 \leq j \leq 2$. It also follows that $L_{-j}=L_{0}$ for every $-1 \leq j \leq 2$ from what was just proved. So as $L_{0}=\lim _{n \rightarrow \infty} \inf x_{n}$, there exists a positive integer $s$ such that $r_{s} \geq 0$ and

$$
\max \left\{x_{r_{s-2}}, x_{r_{s-1}}, x_{r_{s}}, x_{r_{s+1}}\right\} \leq \frac{1}{2}\left(\lim _{n \rightarrow \infty} \sup x_{n}+\lim _{n \rightarrow \infty} \inf x_{n}\right) .
$$

It follows by Lemma 2 that

$$
x_{n} \leq \frac{1}{2}\left(\lim _{n \rightarrow \infty} \sup x_{n}+\lim _{n \rightarrow \infty} \inf x_{n}\right)
$$

for all $n \geq r_{s+1}$. Thus, using the assumption $\lim _{n \rightarrow \infty} \inf x_{n}<\lim _{n \rightarrow \infty} \sup x_{n}$, it follows that

$$
\lim _{n \rightarrow \infty} \sup x_{n} \leq \frac{1}{2}\left(\lim _{n \rightarrow \infty} \sup x_{n}+\lim _{n \rightarrow \infty} \inf x_{n}\right)<\lim _{n \rightarrow \infty} \sup x_{n} .
$$

\section{REFERENCES}

[1] A. M. Amleh, E. Camouzis, and G. Ladas, On the dynamics of a rational difference equation, Part 1, Int. J. Difference Equ., 3 (2008), 1-35.

[2] A. M. Amleh, E. Camouzis, and G. Ladas, On the dynamics of a rational difference equation, Part 2, Int. J. Difference Equ., 3 (2008), 195-225.

[3] A. M. Amleh, E. Camouzis, G. Ladas, and M. Radin, Patterns of boundedness of a rational system in the plane, J. Difference Equ. Appl., 16 (2010), 1197-1236.

[4] I. Bajo, Daniel Franco, and J. Peán, Dynamics of a rational system of difference equations in the plane, Adv. Difference Equ., 2011, Art. ID 958602, 17 pp.

[5] S. Basu and O. Merino, Global behavior of solutions to two classes of second-order rational difference equations, Adv. Difference Equ., 2009, Art. ID 12860227 pp. 
[6] S. Basu and O. Merino, On the global behavior of solutions to a planar system of difference equations, Comm. Appl. Nonlinear Anal., 16 (2009), 89-111.

[7] A. Brett, E. Camouzis, C. Lynd, and G. Ladas, On the boundedness character of a rational system, JNMaS, 1 (2009), 1-10.

[8] E. Camouzis, Boundedness of solutions of a rational system of difference equations, Proceedings of the 14th International Conference on Difference Equations and Applications held in Instanbul, Turkey, July 21-25, 2008, Ugur-Bahcesehir University Publishing Company, Istanbul, Turkey, Difference Equations and Applications, ISBN 978-975-6437-80-3 (2009), 157-164 .

[9] E. Camouzis, E. Drymonis, and G. Ladas, On the global character of the system $x_{n+1}=\frac{\alpha}{x_{n}+y_{n}}$ and $y_{n+1}=\frac{y_{n}}{B x_{n}+y_{n}}$, Commun. Appl. Nonlinear Anal., 16 (2009), $51-64$.

[10] E. Camouzis, E. Drymonis, and G. Ladas, Patterns of boundedness of the rational system $x_{n+1}=\frac{\alpha_{1}+\beta_{1} x_{n}}{A_{1}+C_{1} y_{n}}$ and $y_{n+1}=\frac{\alpha_{2}+\beta_{2} x_{n}+\gamma_{2} y_{n}}{A_{2}+B_{2} x_{n}+y_{n}}$, Fasc. Math., 44 (2010), 9-18.

[11] E. Camouzis, E. Drymonis, G. Ladas, and W. Tikjha, Patterns of boundedness of the rational system $x_{n+1}=\frac{\alpha_{1}}{A_{1}+B_{1} x_{n}+C_{1} y_{n}}$ and $y_{n+1}=\frac{\alpha_{2}+\beta_{2} x_{n}+\gamma_{2} y_{n}}{A_{2}+B_{2} x_{n}+y_{n}}$, J. Difference Equ. Appl., (2011).

[12] E. Camouzis, E. Drymonis, and G. Ladas, Patterns of Boundedness of the Rational System $x_{n+1}=\frac{\alpha_{1}+\beta_{1} x_{n}}{A_{1}+B_{1} x_{n}+C_{1} y_{n}}$ and $y_{n+1}=\frac{\alpha_{2}+\beta_{2} x_{n}+\gamma_{2} y_{n}}{A_{2}+B_{2} x_{n}+C_{2} y_{n}}$, Communications on Applied Nonlinear Analysis, 18 (2011), 1-23.

[13] E. Camouzis, A. Gilbert, M. Heissan, and G. Ladas, On the boundedness character of the system $x_{n+1}=\frac{\alpha_{1}+\gamma_{1} y_{n}}{x_{n}}$ and $y_{n+1}=\frac{\alpha_{2}+\beta_{2} x_{n}+\gamma_{2} y_{n}}{A_{2}+x_{n}+y_{n}}$,Commun. Math. Anal., 7 (2009), 41-50.

[14] E. Camouzis, C. M. Kent, G. Ladas, and C. D. Lynd, On the global character of solutions of the system $x_{n+1}=\frac{\alpha_{1}+y_{n}}{x_{n}}$ and $y_{n+1}=\frac{\alpha_{2}+\beta_{2} x_{n}+\gamma_{2} y_{n}}{A_{2}+B_{2} x_{n}+C_{2} y_{n}}$, J. Difference Equ. Appl., (2011).

[15] E. Camouzis, M. R. S. Kulenović, G. Ladas, and O. Merino, Rational systems in the plane, J. Difference Equ. Appl., 15 (2009), 303-323.

[16] E. Camouzis and G. Ladas, Dynamics of Third-Order Rational Difference Equations; With Open Problems and Conjectures, Chapman \& Hall/CRC Press, November 2007.

[17] E. Camouzis and G. Ladas, Global results on rational systems in the plane, I, J. Difference. Equ. Appl., 16 (2010), 975-1013.

[18] E. Camouzis, G. Ladas, and L. Wu, On the global character of the system $x_{n+1}=$ $\frac{\alpha_{1}+\gamma_{1} y_{n}}{x_{n}}$ and $y_{n+1}=\frac{\beta_{2} x_{n}+\gamma_{2} y_{n}}{B_{2} x_{n}+C_{2} y_{n}}$, Int. J. Pure Appl. Math., 53 (2009), 21-36.

[19] D. Clark and M. R. S. Kulenović, On a coupled system of rational difference equations, Comput. Math. Appl., 43 (2002), 849-867.

[20] D. Clark, M. R. S. Kulenović, and J. F. Selgrade, Global asymptotic behavior of a two dimensional difference equation modelling competition, Nonlinear Anal. TMA, 52 (2003), 1765-1776.

[21] C. A. Clark, M. R. S. Kulenović, and J.F. Selgrade, On a system of rational difference equations, J. Difference Equ. Appl., 11 (2005), 565-580.

[22] A.S. Clark and J. Weiss, On the geometry of a four-parameter rational planar system of difference equations, J. Difference Equ. Appl., 18 (2012), 509-524.

[23] J. M. Cushing, S. Levarge, N. Chitnis and S. M. Henson, Some discrete competition models and the competitive exclusion principle, J. Difference Equ. Appl., 10 (2004), $1139-1152$.

[24] E. Drymonis, Y. Kostrov, and Z. Kudlak, On rational difference equations with nonnegative periodic coefficients, Int. J. Difference Equ., 10 (2012). 
[25] M. Garić-Demirović, M. R. S. Kulenović, and M. Nurkanović, Global behavior of four competitive rational systems of difference equations on the plane, Discrete Dyn. Nat. Soc., 2009, Art. ID 153058, 34 pp.

[26] E. A. Grove, Y. Kostrov, M. A. Radin, and S. Schultz, On the global character of solutions of the system $x_{n+1}=\frac{\alpha_{1}}{x_{n}+y_{n}}$ and $y_{n+1}=\frac{\alpha_{2}+\beta_{2} x_{n}}{B_{2} x_{n}+y_{n}}$, Commun. Appl. Nonlinear Anal., 17 (2010), 69-81.

[27] E. A. Grove and G. Ladas, Periodicities in Nonlinear Difference Equations, Chapman \& Hall/CRC Press, 2005.

[28] S. Kalabusić and M. R. S. Kulenović, Dynamics of certain anti-competitive systems of rational difference equations in the plane, J. Difference Equ. Appl., 17 (2011), $1599-1615$

[29] S. Kalabusić, M. R. S. Kulenović and E. Pilav, Global dynamics of a system of rational difference equations in the plane, Adv. Difference Equ., 2009, Art. ID 132802, 30 pp.

[30] Y. S. Knopf and M. Huang, Global convergence properties of first-order homogeneous systems of rational difference equations, J. Difference Equ. Appl., DOI 10.1080/10236198.2011.590802, 25 pp.

[31] V.L. Kocic and G. Ladas, Global Behavior of Nonlinear Difference Equations of Higher Order with Applications, Kluwer Academic Publishers, Dordrecht, 1993.

[32] M. R. S. Kulenović and O. Merino, Competitive-exclusion versus competitivecoexistence for systems in the plane, Discrete Contin. Dyn. Syst. Ser. B, 6 (2006), 1141-1156.

[33] M. R. S. Kulenović and O. Merino, Invariant manifolds for competitive discrete systems in the plane, Int. J. Bifurcations Chaos, 20 (2010), 2471-2486.

[34] M. R. S. Kulenović, O. Merino and M. Nurkanović, Global dynamics of certain competitive system in the plane, J. Difference Equ. Appl., 2011, DOI 10.1080/10236198.2011.605357, 16 pp.

[35] M. R. S. Kulenović and G. Ladas, Dynamics of Second Order Rational Difference Equations; with Open Problems and Conjectures, Chapman \& Hall/CRC Press, 2001.

[36] M. R. S. Kulenović and M. Nurkanović, Asymptotic behavior of two dimensional linear fractional system of difference equations, Rad. Mat., 11 (2002), 59-78.

[37] M. R. S. Kulenović and M. Nurkanović, Asymptotic behavior of a linear fractional system of difference equations, J. Inequal. Appl., (2005), 127-144.

[38] M. R. S. Kulenović and M. Nurkanović, Asymptotic behavior of a competitive system of linear fractional difference equations, Adv. Difference Equ., 3 (2006), 1-13.

[39] G. Ladas, Open problems and conjectures, J. Difference Equ. Appl., 1 (4) (1995), 413-419.

[40] G. Ladas, G. Tzanetopoulos, and A. Tovbis, On May's host parasitoid model, J. Difference Equ. Appl., 2 (1996), 195-204.

[41] G. Lugo and F. J. Palladino, On a second-order rational difference equation and a rational system, 2012, arXiv:1202.0861v1 .

[42] G. Lugo and F. J. Palladino, On the boundedness character of rational systems in the plane. J. Difference Equ. Appl. (2011), 1-11

[43] G. Lugo and F. J. Palladino, Unboundedness for some classes of rational difference equations, Int. J. Difference Equ., 4 (2009), 97-113.

[44] G. Lugo and F. J. Palladino, Unboundedness results for fourth order rational difference equations, J. Differ. Equ. Appl., 18 (5) (2012), 879-893.

[45] E. Magnucka-Blandzi and J. Popenda, On the asymptotic behavior of a rational system of difference equations, J. Difference Equ. Appl., 5 (3) (1999), 271-286. 
[46] O. Merino, Global attractivity of the equilibrium of a difference equation: an elementary proof assisted by computer algebra system, J. Difference Equ. Appl., 17 (2011), $33-41$.

(Received: May 6, 2012)

University of Rhode Island

Department of Mathematics

Kingston, RI 02881-0816, USA

E-mails: gladas@math.uri.edu glugo@math.uri.edu frank@math.uri.edu 